Pacific Journal of Mathematics

MULITPLICITY TYPE AND CONGRUENCE RELATIONS IN 


\title{
MULTIPLICITY TYPE AND CONGRUENCE RELATIONS IN UNIVERSAL ALGEBRAS
}

\author{
T. P. Whaley
}

For given multiplicity types $\mu$ and $\mu^{\prime}$ we consider the possibility of always replacing a universal algebra $\langle A ; F\rangle$ of multiplicity type $\mu$ with an algebra $\langle A ; G\rangle$ of multiplicity type $\mu^{\prime}$ which has exactly the same congruence relations.

In [1] Gould considered the corresponding problem for subalgebra structures. There he completely determined those types $\mu^{\prime}$ which could replace a given type $\mu$. His results were very positive; e.g., any countable type with finitely many nonzero entries can always be replaced by a type representing a single operation. We do not completely determine which types can replace a given type in the congruence ralation sense, but give necessary conditions which show that simplifications as in the subalgebra case are impossible. We also show that no two finite types are interchangeable with respect to congruence relations.

In this paper we shall be concerned only with the congruence relations of the algebras considered so we may disregard nullary operations. Thus we alter the notion of multiplicity type as follows:

Definition 1.1. By the multiplicity type of an algebra $\mathscr{A}$ we mean the sequence $\mu=\left\langle\mu_{1}, \mu_{2}, \cdots, \mu_{n}, \rightarrow\right\rangle_{n \varepsilon \omega}$ where $\mathscr{A}$ has exactly $\mu_{i}$ operations of rank $i$ for $i=1,2, \cdots$.

DEFINITION 1.2. We denote the set of all congruence relations of the algebra $\mathscr{A}$ by $\Theta(\mathscr{A})$. If $a, b$ are elements of the algebra $\mathscr{A}$, we denote by $\Theta(a, b)$ the smallest congruence relation of $\mathscr{A}$ which contains $(a, b)$.

Definition 1.3. If $\mu=\left\langle\mu_{1}, \mu_{2}, \cdots, \mu_{n}, \rightarrow\right\rangle$ and $\mu^{\prime}=\left\langle\mu_{1}^{\prime}, \mu_{2}^{\prime}, \cdots\right.$, $\left.\mu_{n}^{\prime}, \rightarrow\right\rangle$ are sequences of cardinal numbers, we write $\mu \leqq \mu^{\prime}$ provided for any algebra $\mathscr{A}=\langle A ; F\rangle$ of multiplicity type $\mu$ there is an algeba $\mathscr{A}^{\prime}=\left\langle A ; F^{\prime}\right\rangle$ of multiplicity type $\mu^{\prime}$ such that $\Theta(\mathscr{A})=\Theta\left(\mathscr{A}^{\prime}\right)$.

2. A necessary condition for $\mu \leqq \mu^{\prime}$. The purpose of this section is to prove the following theorem which shows that in considering congruence relations, as contrasted with subalgebras, the number of operations present is very crucial.

THEOREM 2.1. If $\mu \leqq \mu^{\prime}$, then $\Sigma \mu_{i} \leqq \Sigma \mu_{i}^{\prime}$.

To prove the theorem, we construct, for each cardinal $m \geqq 2$, 
an algebra $\mathscr{A}_{m}=\left\langle A_{m}, f_{\xi}\right\rangle_{1 \leqq \xi \leqq m}$ with each $f_{\xi}$ a unary operation and such that $\Theta\left(\mathscr{A}^{\prime}\right) \neq \Theta\left(\mathscr{A}_{m}\right)$ if $\mathscr{A}^{\prime}=\left\langle A_{m}, G\right\rangle$ is of type $\mu^{\prime}$ where $\Sigma \mu_{i}^{\prime}<m$.

To construct $\mathscr{A}_{m}$, let $A_{m}=\left\{a_{\xi}: 0 \leqq \xi \leqq m\right\} \cup\left\{b_{\xi}: 0 \leqq \xi \leqq m\right\}$ where the $a_{\xi}^{\prime} \mathrm{S}$ and $b_{\xi}^{\prime} \mathrm{S}$ are all distinct. Let $B_{m}=A_{m} \sim\left\{a_{0}, b_{0}\right\}$. Now if $1 \leqq \xi \leqq m$ let $f_{\xi}$ be defined by $f_{\xi}\left(a_{0}\right)=a_{\xi}, f_{\xi}\left(b_{0}\right)=b_{\xi}$, and $f_{\xi}(x)=x$ if $x \in B_{m}$. Then $\mathscr{A}_{m}=\left\langle A_{m} ; f_{\xi}\right\rangle_{1 \leqq \vdots \leqq m}$.

Lemma 2.2. Letting $i d_{x}$ denote the identity relation on $X$ we have

(i) if $x, y \in B_{m}$, then $\theta(x, y)=i d_{A_{m}} \cup\{(x, y\},(y, x)\}$,

(ii) $\theta\left(a_{0}, b_{0}\right)=i d_{A_{m}} \cup\left\{\left(a_{\xi}, b_{\xi}\right): 0 \leqq \xi \leqq m\right\} \cup\left\{\left(b_{\xi}, a_{\xi}\right): 0 \leqq \xi \leqq m\right\}$

(iii) if $x \in B_{m}$, then

$$
\theta\left(a_{0}, x\right)=i d_{A_{m}} \cup\left[\{x\} \cup\left\{a_{\xi}: 0 \leqq \xi \leqq m\right\}\right]^{2}
$$

and

$$
\theta\left(b_{0}, x\right)=i d_{A_{m}} \cup\left[\{x\} \cup\left\{b_{\xi}: 0 \leqq \xi \leqq m\right\}\right]^{2} .
$$

Proof. Both (i) and (ii) are clear. For (iii) we consider $\theta\left(a_{0}, x\right)$. Now since $\left(a_{0}, x\right) \in \theta\left(a_{0}, x\right)$ we have $\left(f_{\xi}\left(a_{0}\right), f_{\xi}(x)\right)=\left(a_{\xi}, x\right) \in \theta\left(a_{0}, x\right)$ for each $\xi$. By transitivity we get $\left(a_{\xi}, a_{\eta}\right) \in \theta\left(a_{0}, x\right)$ for each $\xi, \eta$ with $1 \leqq \xi \leqq m$ and $1 \leqq \eta \leqq m$. Thus $\left[\{x\} \cup\left\{a_{\xi}: 0 \leqq \xi \leqq m\right\}\right]^{2} \leqq \theta\left(a_{0}, x\right)$. Since $i d_{A_{m}} \cup\left[\{x\} \cup\left\{a_{\xi}: 0<\xi \leqq m\right\}\right]^{2}$ is a congruence relation, the proof is completed. The claim for $\theta\left(b_{0}, x\right)$ follows by symmetry.

LEMMA 2.3. Let $f$ be a unary operation on $A_{m}$ which preserves the congruence relations of $\mathscr{A}_{m}$ (i.e. adding $f$ as an operation would not affect the congruence relations). Then $f \mid B_{m}=i d_{B_{m}}$ or $f \mid B_{m}$ is constant.

Proof. Let us assume that $f \mid B_{m} \neq i d_{B_{m}}$. Then there is some $x \in B_{m}$ such that $f(x)=y \neq x$. Suppose $y \in B_{m}$. Let $z \in B_{m}, z \notin\{x, y\}$. Then $(f(z),(f(x))=(f(z), y) \in \theta(x, z)$. By Lemma 2.2. part (i) we get $f(z)=y$. Also we have $(f(x), f(z))=(f(z), f(y))=(y, f(y)) \in \theta(x, y) \cap$ $\theta(z, y)$. Thus $f(y)=y$ and $f \mid B_{m}$ is constant.

Now if $y \notin B_{m}$, we have for any $z \in B_{m}$ that $(f(x), f(z))=(y, f(z)) \in$ $\theta(x, z)$ so $f(z)=y$ and $f \mid B_{m}$ is constant.

LEMMA 2.4. Let $f$ be a unary operation on $A_{m}$ which preserves the congruence relations of $\mathscr{A}_{m}$. Then if $f \mid B_{m}$ is constant, $f$ is constant.

Proof. Suppose first that $f \mid B_{m} \equiv b$ for some $b \in B_{m}$. Without loss of generality we may assume that $f \mid B_{m} \equiv a_{2}$. Then $\left(f\left(a_{1}\right), f\left(b_{0}\right)\right)=$ $\left(a_{2}, f\left(b_{0}\right)\right) \in \theta\left(a_{1}, b_{0}\right)$ so by Lemma 2.3. (iii) we have $f\left(b_{0}\right)=a_{2}$. Also, 
$\left(f\left(a_{0}\right), f\left(a_{2}\right)\right)=\left(f\left(a_{0}\right), a_{2}\right) \in \theta\left(a_{0}, a_{2}\right)$ so $f\left(a_{0}\right) \in\left\{a_{\xi} \mid 0 \leqq \xi \leqq m\right\}$. Furthermore $\left(f\left(a_{0}\right), f\left(b_{0}\right)\right)=\left(f\left(a_{0}\right), a_{2}\right) \in \theta\left(a_{0}, b_{0}\right)$ giving $f\left(a_{0}\right)=a_{2}$.

Now assume that $f \mid B_{m} \equiv a_{0}$ (the case $f / B_{m} \equiv b_{0}$ follows by symmetry). Then $\left(f\left(a_{1}\right), f\left(a_{0}\right)\right)=\left(a_{0}, f\left(a_{0}\right)\right) \in \theta\left(a_{1}, a_{0}\right)$ so $f\left(a_{0}\right) \in\left\{a_{\xi} \mid 0 \leqq \xi \leqq m\right\}$. Also we have $\left(f\left(b_{1}\right), f\left(b_{0}\right)\right)=\left(a_{0}, f\left(b_{0}\right)\right) \in \theta\left(b_{1}, b_{0}\right)$ so $f\left(b_{0}\right)=a_{0}$. Finally $\left(f\left(b_{0}\right), f\left(a_{0}\right)\right)=\left(a_{0}, f\left(a_{0}\right)\right) \in \theta\left(b_{0}, a_{0}\right)$ so $f\left(a_{0}\right)=a_{0}$.

LEMMA 2.5. If $f$ is a unary operation on $A_{m}$ which preserves the congruence relations of $\mathscr{A}_{m}$, then $f=i d_{A_{m}}, f$ is constant, or $f=f_{\xi}$ for some $\xi, 1 \leqq \xi \leqq m$.

Proof. Assume that $f$ is not constant. By Lemmas 2.3 and 2.4 we know that $f \mid B_{m}=i d_{B_{m}}$. Thus $\left(f\left(b_{1}\right), f\left(b_{0}\right)\right)=\left(b_{1}, f\left(b_{0}\right)\right) \in \theta\left(b_{1}, b_{0}\right)$ so $f\left(b_{0}\right) \in\left\{b_{\xi} \mid 0 \leqq \xi \leqq m\right\}$. Similarly $f\left(a_{0}\right) \in\left\{a_{\xi} \mid 0 \leqq \xi \leqq m\right\}$. Since $\left(f\left(a_{0}\right), f\left(b_{0}\right)\right) \in \theta\left(a_{0}, b_{0}\right)$, we know that for some $\xi, 0 \leqq \xi \leqq m$, we have $\left(f\left(a_{0}\right), f\left(b_{0}\right)\right)=\left(a_{\xi}, b_{\xi}\right)$.

Notation. Suppose $g$ is an $n$-ary operation on the set $X$. If $1 \leqq k \leqq n$, if $c_{1}, \cdots, c_{n-k} \in X$, if $\{1, \cdots, n\}=\left\{i_{1}, \cdots, i_{n-k}\right\} \cup\left\{j_{1}, \cdots, j_{k}\right\}$, then we denote by $g\left[i_{1}, \cdots, i_{n-k} ; c_{1}, \cdots, c_{n-k}\right]$ the $k$-ary operation on $X$ defined by

$$
g\left[i_{1}, \cdots, i_{n-k} ; c_{1}, \cdots, c_{n-k}\right]\left(x_{1}, \cdots, x_{k}\right)=g\left(y_{1}, \cdots, y_{n}\right)
$$

where

$$
y_{j}=\left\{\begin{array}{lll}
c_{s} & \text { if } & j=i_{s} \\
x_{j} & \text { if } & j=j_{s}
\end{array}\right.
$$

More informally $g\left[i_{1}, \cdots, i_{n-k} ; c_{1}, \cdots, c_{n-k}\right]$ is obtained by holding each $c_{j}$ fixed in the $i_{j}$ coordinate of $g$.

REMARK. An operation preserves the congruence relations of an algebra if and only if each of its unary translations preserves the congruence relations of the algebra. Thus if $g$ is an operation on $A_{m}$ which preserves the congruence relations of $\mathscr{A}_{m}$, then a given unary translation of $g$ must be the identity map, a constant map or else one of the $f_{\xi}$. It is the purpose of the next lemma to show that only one $f_{\xi}$ can be so obtained from a given operation $g$.

LEMMA 2.6. Let $g$ be an n-ary operation of $A_{m}$ which preserves the congruence relations of $\mathscr{A}_{m}$. If $g\left[i_{1}, \cdots, i_{n-1} ; c_{1}, \cdots, c_{n-1}\right]=f_{\xi}$ for some $i_{1}, \cdots, i_{n-1} ; c_{1}, \cdots, c_{n-1}, \xi$ where $1 \leqq i_{j} \leqq n, c_{j} \in A_{m}$, and $1 \leqq \xi \leqq m$, then $g\left[i_{1}, \cdots, i_{n-1} ; d_{1}, \cdots, d_{n-1}\right]=f_{\xi}$ for each $d_{1}, \cdots, d_{n-1} \in A_{m}$. 
Proof. The proof is by induction on $n$. The first case to consider is $n=2$. Without loss of generality we assume that $g[1, c]=f_{1}$ and show that $g[1, d]=f_{1}$ for each $d \in A_{m}$. We consider the cases $c=a_{1}$, $c=a_{0}$, and $c=a_{2}$. The case $c=a_{j}$ with $j>2$ would be handled just as $c=a_{2}$, and $c=b_{i}$ would be symmetric to $c=a_{i}$.

Case 1. $c=a_{1}$ : We are assuming now that $g\left(a_{1}, x\right)=f_{1}(x)$ for all $x \in A_{m}$. Since $\left(g\left(b_{1}, a_{0}\right), g\left(a_{1}, a_{0}\right)\right)=\left(g\left(b_{1}, a_{0}\right), a_{1}\right) \in \theta\left(b_{1}, a_{1}\right)$, we have $g\left(b_{1}, a_{0}\right)=a_{1}$ or $g\left(b_{1}, a_{0}\right)=b_{1}$. If $g\left(b_{1}, a_{0}\right)=b_{1}$, then $g\left[1, b_{1}\right] \equiv b_{1}$. But this would give $\left(g\left(b_{1}, a_{2}\right), g\left(a_{1}, a_{2}\right)=\left(b_{1}, a_{2}\right)\right) \in \theta\left(b_{1}, a_{1}\right)$, a contradiction. Thus $g\left(b_{1}, a_{0}\right)=a_{1}$. From this we see that $g\left[1, b_{1}\right]=f_{1}$ or $g\left[1, b_{1}\right] \equiv a_{1}$. However, if $g\left[1, b_{1}\right] \equiv a_{1}$, then $\left(g\left(b_{1}, a_{2}\right), g\left(a_{1}, a_{2}\right)\right)=\left(a_{1}, a_{2}\right) \in \theta\left(b_{1}, a_{1}\right)$, a contradiction. Therefore, $g\left[1, b_{1}\right]=f_{1}$.

Now since $\left(g\left(a_{0}, b_{0}\right), g\left(a_{1}, b_{0}\right)\right)=\left(g\left(a_{0}, b_{0}\right), b_{1}\right) \in \theta\left(a_{0}, a_{1}\right)$ gives $g\left(a_{0}, b_{0}\right)=b_{1}$, we have $g\left[1, a_{0}\right] \equiv b_{1}$ or $g\left[1, a_{0}\right]=f_{1}$. Noting that $\left(g\left(a_{0}, a_{0}\right), g\left(a_{1}, a_{0}\right)\right)=$ $\left(g\left(a_{0}, a_{0}\right), a_{1}\right) \in \theta\left(a_{0}, a_{1}\right)$ we see that $g\left(a_{0}, a_{0}\right) \in\left\{a_{\xi} \mid 0 \leqq \xi \leqq m\right\}$. Thus $g\left[1, a_{0}\right] \neq b_{1}$ so $g\left[1, a_{0}\right]=f_{1}$. By symmetry we get $g\left[1, b_{0}\right]=f_{1}$.

For $2 \leqq \xi \leqq m$ we have $\left(g\left(a_{\xi}, b_{0}\right), g\left(a_{1}, b_{0}\right)\right)=\left(g\left(a_{\xi}, b_{0}\right), b_{1}\right) \in \theta\left(a_{\xi}, a_{1}\right)$ so $g\left(a_{\xi}, b_{0}\right)=b_{1}$. Thus $g\left[1, a_{\xi}\right] \equiv b_{1}$ or $g\left[1, a_{\xi}\right]=f_{1}$. Now $\left(g\left(a_{\xi}, a_{1}\right)\right.$, $\left.g\left(a_{1}, a_{1}\right)\right)=\left(g\left(a_{\xi}, a_{1}\right), a_{1}\right) \in \theta\left(a_{\xi}, a_{1}\right)$ so $g\left(a_{\xi}, a_{1}\right) \neq b_{1}$. Thus $g\left[1, a_{\xi}\right]=f_{1}$, and by symmetry $g\left[1, b_{\xi}\right]=f_{1}$.

Case 2. $c=a_{0}$ : We are assuming that $g\left(a_{0}, x\right)=f_{1}(x)$ for each $x \in A_{m}$. Thus we have $\left(g\left(a_{1}, b_{0}\right), g\left(a_{0}, b_{0}\right)\right)=\left(g\left(a_{1}, b_{0}\right), b_{1}\right) \in \theta\left(a_{1}, a_{0}\right)$. Hence $g\left(a_{1}, b_{0}\right)=b_{1} \quad$ so $\quad g\left[1, a_{1}\right] \equiv b_{1} \quad$ or $g\left[1, a_{1}\right]=f_{1}$. If $g\left[1, a_{1}\right] \equiv b_{1}$, then $\left(g\left(a_{1}, a_{2}\right), g\left(a_{0}, a_{2}\right)\right)=\left(b_{1}, a_{2}\right) \in \theta\left(a_{1}, a_{0}\right)$, a contradiction. Thus $g\left[1, a_{1}\right]=f_{1}$, and we have Case 1.

Case 3. $c=a_{2}$ : Now we are assuming that $g\left(a_{2}, x\right)=f_{1}(x)$ for each $x \in A_{m}$. Here we have $\left(g\left(a_{0}, b_{0}\right), g\left(a_{2}, b_{0}\right)\right)=\left(g\left(a_{0}, b_{0}\right), b_{1}\right) \in \theta\left(a_{0}, a_{2}\right)$ so $g\left(a_{0}, b_{0}\right)=b_{1}$. Thus $g\left[1, a_{0}\right] \equiv b_{1}$ or $g\left[1, a_{0}\right]=f_{1}$. If $g\left[1, a_{0}\right] \equiv b_{1}$, then $\left(g\left(a_{0}, a_{2}\right), g\left(a_{2}, a_{2}\right)\right)=\left(b_{1}, a_{2}\right) \in \theta\left(a_{0}, a_{2}\right)$, a contradiction. Therefore, $g\left[1, a_{0}\right]=f_{1}$, and we have Case 2 . This completes the step $n=2$ in the induction argument.

Let us assume that the lemma holds for $n=k$ and that $g$ is $(k+1)$ ary with $g\left[i_{1}, i_{2}, \cdots, i_{k} ; c_{1}, \cdots, c_{k}\right]=f_{\xi^{\circ}} \quad$ Without loss of generality we take $g\left[1,2, \cdots, k ; c_{1}, \cdots, c_{k}\right]=f_{\xi}$. Thus $g\left(c_{1}, \cdots, c_{k}, x\right)=f_{\hat{\xi}}(x)$ for all $x \in A$. Applying the induction hypothesis to the $k$-ary operation $g\left[1, c_{1}\right]$ we get $g\left(c_{1}, d_{2}, d_{3}, \cdots, d_{k}, x\right)=f_{\xi}(x)$ for all $d_{j} \in A_{m}$ and all $x \in A_{m}$. Now we apply the case $n=2$ to the binary operation $g\left[2,3, \cdots, k ; d_{2}, d_{3}\right.$, $\left.\cdots, d_{k}\right]$ to get $g\left(d_{1}, d_{2}, \cdots, d_{k}, x\right)=f_{\xi}(x)$ for arbitrary elements $d_{1}, \cdots, d_{k}$.

COROLlaRY 2.7. Let $g$ be an n-ary operation on $A_{m}$ which preserves the congruence relations of $\mathscr{A}_{m}$. Then all nonconstant, nonidentity unary translations of $g$ are equal. 
Proof. Suppose $g\left[i_{1}, \cdots, i_{n-1} ; c_{1}, \cdots, c_{n-1}\right]=f_{\xi}$. By Lemma 2.6 any unary translation of $g$ obtained by fixing these same coordinates is equal to $f_{\xi}$. Let $\{1, \cdots, n\}=\left\{i_{1}, \cdots, i_{n-1}, j\right\}$. Then for any $x_{1}, \cdots$, $x_{n} \in A_{m}$ we have

$$
\begin{aligned}
g\left(x_{1}, \cdots, x_{n}\right) & =g\left[i_{1}, \cdots, i_{n-1} ; y_{1}, \cdots, y_{n-1}\right]\left(x_{j}\right) \\
& =f_{\xi}\left(x_{j}\right)
\end{aligned}
$$

where $y_{k}=x_{i_{k}}$ for $k=1, \cdots, n-1$.

Now consider a unary translation of $g$ obtained by fixing another set of $n-1$ coordinates; say $g\left[j_{1}, \cdots, j_{n-1} ; d_{1}, \cdots, d_{n-1}\right]$ where $j=j_{k}$ for some $k, 1 \leqq k \leqq n-1$. Then for any $x \in A_{m}$

$$
\begin{aligned}
g\left[j_{1}, \cdots, j_{n-1} ; d_{1}, \cdots, d_{n-1}\right](x) & =g\left(y_{1}, \cdots, y_{n}\right) \\
& =f_{\xi}\left(y_{j}\right) \\
& =f_{\xi}\left(y_{j_{k}}\right) \\
& =f_{\xi}\left(d_{k}\right)
\end{aligned}
$$

where

$$
y_{i}=\left\{\begin{array}{lll}
d_{s} & \text { if } & i=j_{s} \\
x & \text { if } & i \notin\left\{j_{1}, \cdots, j_{n-1}\right\}
\end{array} .\right.
$$

Thus $g\left[j_{1}, \cdots, j_{n-1} ; d_{1}, \cdots, d_{n-1}\right]$ is constant.

Proof of Theorem 2.1. Suppose $\mu$ and $\mu^{\prime}$ are sequences with $\Sigma \mu_{i}^{\prime}<$ $\Sigma \mu_{i}=m$. Clearly $\mu \geqq \varepsilon_{m}=\langle m, 0,0, \cdots\rangle$. Since $\geqq$ is transitive, it is enough to show that $\mu^{\prime} \geqq \varepsilon_{m}$. Now $\mathscr{A}_{m}$ is an algebra of type $\varepsilon_{m}$. Suppose $\mathscr{A}=\left\langle A_{m} ; G\right\rangle$ is an algebra of type $\mu^{\prime}$ such that $\Theta(\mathscr{A})=\Theta\left(\mathscr{A}_{m}\right)$. Then $\Theta\left(\mathscr{A}_{m}\right)=\Theta(\mathscr{\mathscr { A }})$ where $\overline{\mathscr{A}}=\left\langle A_{m} ; \bar{G}\right\rangle$ with $\bar{G}$ consisting of the unary translations of $G$. Now by Lemma 2.5 each element of $\bar{G}$ is the identity, a constant, or one of the $f_{\xi}^{\prime}$ s. By Lemma 2.6 at most $\Sigma \mu_{i}^{\prime}$ of the $f_{\xi}^{\prime} \mathrm{S}$ can be so obtained. Let $\eta$ be such that $f_{\eta} \notin \bar{G}$. Then $\left(a_{\eta}, b_{\eta}\right) \notin \theta\left(a_{0}, b_{0}\right)$ in $\overline{\mathscr{A}}$. This contradiction shows that $\mu^{\prime} \supsetneqq \varepsilon_{m}$ and completes the proof of Theorem 2.1.

\section{Finite Types.}

Definition 3.1. A sequence $\mu$ is said to be finite if $\Sigma \mu_{i}$ is finite.

Notation. For a finite sequence $\mu$, we let $l(\mu)=n$ if $n$ is the largest interger such that $\mu_{n} \neq 0$.

Lemma 3.2. If $\mathscr{D}=\langle D ; F\rangle$ is an algebra of finite multiplicity type $\mu$ and if $C=\left\{c_{1}, \cdots, c_{n}\right\}$ is a finite subset of $D$, then the number 
of translations of the operations of $F$ which can be obtained by fixing only elements from $C$ is at most

where

$$
P(\mu, n)=\sum_{k=0}^{l(\mu)-1}\left(\sum_{i=k+1}^{l(\mu)}\left(\begin{array}{l}
i \\
k
\end{array}\right) \mu_{i}\right)^{n^{k}}
$$

$$
\left(\begin{array}{l}
i \\
k
\end{array}\right)=\frac{i !}{k !(i-k) !}
$$

Proof. Suppose $1 \leqq j<k \leqq l(\mu)$ and $f \in F$ is $k$-ary. By fixing $j$ elements from $C$ in $j$ of the coordinates of $f$ we obtain a $(k-j)$-ary translation of $f$. There are $\left(\begin{array}{l}k \\ j\end{array}\right)$ ways to choose the $j$ coordinates and $n^{j}$ ways to choose the constants. Thus for fixed $j$ and $k$ we get $\mu_{k} \cdot\left(\begin{array}{c}k \\ j\end{array}\right) \cdot n^{j}$ translations in this way. For a fixed $j$ we then obtain $\sum_{k=j+1}^{l(k)}\left(\begin{array}{c}k \\ j\end{array}\right) \mu_{k}^{n^{k}}$ translations from $F$ by fixing exactly $j$ elements from $C$. Now summing on $j$ we obtain the desired number of translations.

For a fixed sequence $\mu$ and a fixed nonnegative integer $k$ we now construct an algebra $\mathscr{D}=\langle D ; F\rangle$ of multiplicity type $\mu$ whose congruence relations can not be realized by operations of type $\mu^{\prime}$ if $P\left(\mu^{\prime}, k\right)<$ $P(\mu, k)$. Let $m=P(\mu, k)$, and recall from $\S 2$ that $\mathscr{A}_{m}=\left\langle A_{m} ; f_{\xi}\right\rangle_{1<\xi<m}$. Let $C=\left\{c_{1}, \cdots, c_{k}\right\}$ be such that $c_{i}^{\prime}$ s are distinct and $C \cap A_{m}=\phi$. Now we take $D=C \cup A_{m}$. If $g$ is an operation on $D$, by a $C$ translation of $g$ we mean a translation of $g$ obtained by fixing some of the coordinates of $g$ with elements from $C$. Now any application of $g$ to elements of $D$ may be regarded in a unique way as either an application of $g$ to elements of $C$ or else as an application of a $C$ translation of $g$ to elements of $A_{m}$ (including as a $C$-translation $g$ itself). We thus define $F$ by telling what the elements of $F$ do to elements of $C$ and telling what the $C$-translations of elements of $F$ do to elements of $A_{m}$. If $f \in F$ and $F$ has rank $l$, then we shall have $f\left(c_{i_{1}}, \cdots, c_{i_{l}}\right)=c_{i_{l}}$ if each $c_{i_{j}} \in C$. Now by Lemma 3.2 there will be at most $m C$-translations of elements of $F$. Let us denote these $C$-translations by $\left\{g_{\xi} \mid 1 \leqq \xi \leqq m\right\}$. Now if $g_{\xi}$ is $j$-ary and $x_{s} \in A_{m}$ for $1 \leqq s \leqq j$, then we take $g_{\hat{\xi}}\left(x_{1}, \cdots, x_{j}\right)=f_{\xi}\left(x_{j}\right)$.

The following lemma is clear from the construction of $\mathscr{D}$.

Lemma 3.3. If $\theta \in \Theta\left(\mathscr{A}_{m}\right)$, then $\theta \cup i d_{C} \in \Theta(\mathscr{D})$. Conversely, if $\theta \subseteq\left(A_{m}\right)^{2}$ and if $\theta \cup i d_{C} \in \Theta(\mathscr{D})$, then $\theta \in \Theta\left(\mathscr{A}_{m}\right)$.

LEMMA 3.4. If $g$ is an n-ary operation on $D$ which preserves the congruence relations of $\mathscr{D}$ and if $g\left(x_{1}, \cdots, x_{n}\right)=c \in C$ for some 
$x_{1}, \cdots, x_{n} \in A_{m}$, then $g\left(y_{1}, \cdots, y_{n}\right)=c$ for all $y_{1}, \cdots, y_{n} \in A_{m}$.

Proof. The pair $\left(g\left(x_{1}, \cdots, x_{n}\right), g\left(y_{1}, \cdots, y_{n}\right)\right)$ is in the congruence relation of $\mathscr{D}$ generated by $\left\{\left(x_{1}, y_{1}\right), \cdots,\left(x_{n}, y_{n}\right)\right\}$. But by Lemma 3.3 this congruence relation is contained in $\left(A_{m}\right)^{2} \cup i d_{C}$.

Lemma 3.5. Let $g$ be an n-ary operation on $D$ which preserves the congruence relations of $\mathscr{D}$. If $h$ is a unary translation of $g$, then $h\left|A_{m}=i d_{A_{m}}, h\right| A_{m}$ is constant, or $h \mid A_{m}=f_{\xi}$ for some $\xi, 1 \leqq \xi \leqq m$.

Proof. By Lemma 3.4 if $h \mid A_{m}$ is not constant, then $\left(h \mid A_{m}\right)$ : $A_{m} \rightarrow A_{m}$ and thus $h \mid A_{m}$ preserves the congruence relations of $\mathscr{A}_{m}$. The conclusion now follows from Lemma 2.5 .

Lemma 3.6. Let $g$ be an n-ary operation on $D$ which preserves the congruence relations of $\mathscr{D}$. Then there is at most one $\xi$ such that $f_{\xi}$ is a unary translation of $g \mid\left(A_{m}\right)^{n}$.

Proof. This follows from Lemma 3.3 and Lemma 2.6.

THEOREM 3.7. If $\mu$ and $\mu^{\prime}$ are finite sequences such that $\mu<\mu^{\prime}$, then $P(\mu, k) \leqq P\left(\mu^{\prime}, k\right)$ for each nonnegative integer $k$.

Proof. Suppose $P\left(\mu^{\prime}, k\right)<P(\mu, k)$. The algebra $\mathscr{D}$ is of multiplicity type $\mu$. Suppose $\mathscr{D}^{\prime}=\langle D ; G\rangle$ is of multiplicity type $\mu^{\prime}$ and that $\Theta(\mathscr{D})=\Theta\left(\mathscr{D}^{\prime}\right)$. Let $\bar{G}$ be the set of all $C$-translations of elements of $G$ (again including the elements of $G$ ). Then $\Theta(\mathscr{D})=\Theta\left(\mathscr{D}{ }^{\prime \prime}\right)$ where $\mathscr{D}^{\prime \prime}=\langle D ; \bar{G}\rangle$. Now in $\mathscr{D}$ we have

$$
\left.\theta\left(a_{0}, b_{0}\right)=i d_{D} \cup\left\{a_{i}, b_{i}\right) \mid 1<i \leqq m\right\}^{2} .
$$

Thus for each $i, 1 \leqq i \leqq m$, we must have $g \in \bar{G}$ such that some unary translation of $g$ is $f_{i}$. However, by Lemma 3.6, there is at most one $i$ for a given $g \in \bar{G}$. Furthermore, by Lemma 3.2, the number of elements in $\bar{G}$ is $P(\mu, k)<P(\mu, k)=m$. This contradiction shows that such a $G$ does not exist and thus concludes the proof.

Remark. For a fixed finite sequence $\mu, P(\mu, n)$ is a polynomial in $n$ of degree $l(\mu)-1$ having positive coefficients. The coefficient of $n^{k}$ in $P(\mu, n)$ is $\sum_{i=k+1}^{l(k)}\left(\begin{array}{l}i \\ k\end{array}\right) \mu_{i}$. Hence the following corollaries follow easily from Theorem 3.7 .

COROLLARY 3.8. If $\mu$ and $\mu^{\prime}$ are finite sequences such that $\mu \leqq \mu^{\prime}$ then $l(\mu) \leqq l\left(\mu^{\prime}\right)$. 
COROLLARY 3.9. If $\mu$ and $\mu^{\prime}$ are finite sequences such that $\mu<\mu^{\prime}$ and if $n$ is the largest integer for which $\mu_{n} \neq \mu_{n}^{\prime}$, then $\mu_{n}<\mu_{n}^{\prime}$.

COROLLARY 3.10. If $\mu$ and $\mu^{\prime}$ are finite sequences, then $\mu \nsupseteq \mu^{\prime}$, $\mu^{\prime} \not \mu$, or $\mu=\mu^{\prime}$. Thus among finite types, $\leqq$ is a partial ordering.

REMARK. While a complete characterization of the relation $\leqq$ such as that given by Gould for the case of subalgebras would be of interest, it seems that the results given here indicate that such a result would not be as easily applied as is the subalgebra result. For example, in general we can not reduce the number of operations even by increasing rank.

\section{REFERENCE}

1. M. Gould, Multiplicity type and subalgebra structure in universal algebras, Pacific J. Math. 26 (1968), 469-485.

Received July 15, 1970. This work was partially supported by an NSF COSIP grant. SOUTHWESTERN AT MEMPHIS 


\section{PACIFIC JOURNAL OF MATHEMATICS}

\section{EDITORS}

H. SAMELSON

Stanford University

Stanford, California 94305

C. R. HobBY

University of Washington

Seattle, Washington 98105
J. DugundjI

Department of Mathematics

University of Southern California

Los Angeles, California 90007

RICHARD ARENS

University of California

Los Angeles, California 90024

\section{ASSOCIATE EDITORS}

E. F. BECKENBACH

B. H. NeUMaNN

F. WOLF

K. YoshidA

\section{SUPPORTING INSTITUTIONS}

UNIVERSITY OF BRITISH COLUMBIA

CALIFORNIA INSTITUTE OF TECHNOLOGY

UNIVERSITY OF CALIFORNIA

MONTANA STATE UNIVERSITY

UNIVERSITY OF NEVADA

NEW MEXICO STATE UNIVERSITY

OREGON STATE UNIVERSITY

UNIVERSITY OF OREGON

OSAKA UNIVERSITY
UNIVERSITY OF SOUTHERN CALIFORNIA STANFORD UNIVERSITY

UNIVERSITY OF TOKYO

UNIVERSITY OF UTAH

WASHINGTON STATE UNIVERSITY UNIVERSITY OF WASHINGTON

$\stackrel{*}{*} \stackrel{*}{*} \stackrel{*}{*}$ AMERICAN MATHEMATICAL SOCIETY
NAVAL WEAPONS CENTER 


\section{Pacific Journal of Mathematics}

Vol. 39 , No. 1

May, 1971

Charles A. Akemann, A Gelfand representation theory for $C^{*}$-algebras ....

Sorrell Berman, Spectral theory for a first-order symmetric system of

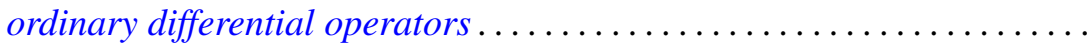

Robert L. Bernhardt, III, On splitting in hereditary torsion theories ........

J. L. Brenner, Geršgorin theorems, regularity theorems, and bounds for determinants of partitioned matrices. II. Some determinantal identities ..........................................

Robert Morgan Brooks, On representing $F^{*}$-algebras .............. 51

Lawrence Gerald Brown, Extensions of topological groups........... 71

Arnold Barry Calica, Reversible homeomorphisms of the real line ........ 79

J. T. Chambers and Shinnosuke Oharu, Semi-groups of local Lipschitzians in

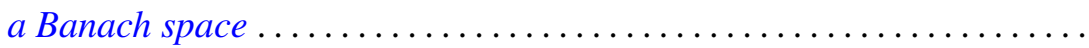

Thomas J. Cheatham, Finite dimensional torsion free rings .............

Byron C. Drachman and David Paul Kraines, A duality between

transpotence elements and Massey products ...................

Richard D. Duncan, Integral representation of excessive functions of a

Markov process ......................................

George A. Elliott, An extension of some results of Takesaki in the reduction

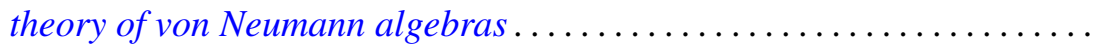

Peter C. Fishburn and Joel Spencer, Directed graphs as unions of partial

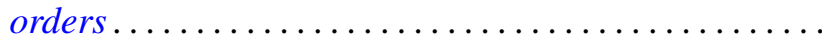

Howard Edwin Gorman, Zero divisors in differential rings ...

Maurice Heins, A note on the Löwner differential equations...

Louis Melvin Herman, Semi-orthogonality in Rickart rings. .

David Jacobson and Kenneth S. Williams, On the solution of linear G.C.D.

equations

Michael Joseph Kallaher, On rank 3 projective planes ... . .

Donald Paul Minassian, On solvable $O^{*}$-groups ...........

Nils Øvrelid, Generators of the maximal ideals of $A(\bar{D})$

Mohan S. Putcha and Julian Weissglass, A semilattice decomposition into

semigroups having at most one idempotent ............

Robert Raphael, Rings of quotients and $\pi$-regularity ....

J. A. Siddiqi, Infinite matrices summing every almost periodic sequence. .

Raymond Earl Smithson, Uniform convergence for multifunctions ...

Thomas Paul Whaley, Mulitplicity type and congruence relations in

universal algebras...

Roger Allen Wiegand, Globalization theorems for locally finitely generated modules... 